\title{
Reutilización de las Aguas Residuales en la Irrigación de Plantas y en la Recuperación de los Suelos
}

\author{
Kelly C. Passarini ${ }^{(1)}$, Felix M. C. Gamarra ${ }^{(2)}$, Rosângela M. Vanalle ${ }^{(1)}$ y José C. C. Santana $a^{(1)}$ \\ (1) Programa de Mestrado em Engenharia de Produção, Universidade Nove de Julho (UNINOVE), \\ Av. Francisco Matarazzo, 612, CEP: 05001-100, São Paulo-Brasil. (e-mail: jccurvelo@uninove.br) \\ (2) Faculdade UNB-Gama, Universidade de Brasília, Área Especial 2, Lote 14 Setor Central, \\ CEP: 72405-610, Gama-DF-Brasil (e-mail: fcarbajal@unb.br)
}

Recibido Mar. 10, 2011; Aceptado Abr. 28, 2011; Versión Final recibida May. 30, 2011

\section{Resumen}

Se presenta un estudio sobre la reutilización de aguas residuales residenciales en la agricultura. Estas aguas residuales conjuntamente con los residuos sólidos de la construcción civil (RCC) fueron tratadas por decantación y por el método de membranas filtrantes. El lodo decantado proveniente del uso del RCC fue utilizado en la producción de humus. El líquido sobrenadante de la decantación fue tratado por membranas filtrantes y utilizado en la irrigación de suelos recién sembrados. Se observó una germinación rápida del 100\% de las semillas, así como una recuperación uniforme del suelo tratado con el humus producido. También se observó una germinación superior al $87 \%$ usando el agua reutilizada. En este contexto, los resultados indican que es posible reutilizar los RCC y las aguas residuales residenciales.

Palabras clave: recuperación de suelos, aguas residuales, residuos da constricción civil, membranas, decantación

\section{Sewage Reuse in Plant Irrigation and Soil Recovery}

\begin{abstract}
A study on the reuse of sewage from homes and buildings in agriculture is presented. These wastewaters together with the compound obtained for construction building waste (CBW) were treated by decantation and by membrane separation processes. The resulting sludge produced by the CBW was used for the production of humus and supernatant water was treated by filtering membrane and reused in irrigation of soils recently seeded. It was observed $100 \%$ of seed germination, with fast and uniform growth in soil containing the produced humus. Also, it was observed germination of over $87 \%$ using reuse water. In this context, the results show that is possible to reuse domestic sewage and CBW.
\end{abstract}

Keywords; reuse, soil recovery, sewage, construction building waste, membrane, decantation. 


\section{INTRODUCCIÓN}

Se estima que el conjunto de procesos que forman la cadena productiva en la construcción civil, es responsable por el $50 \%$ del consumo de todos los recursos naturales disponibles (renovables y los no renovables). También, se estima que los impactos ambientales generados a lo largo de la industria civil son significativos. Entre estos impactos tenemos: el drenaje urbano, la compactación, el comprometimiento de los paisajes naturales, así como la contaminación del suelo, del aire, del agua, entre otros. Diversos estudios, direccionan las investigaciones para la reutilización de los residuos generados por la construcción civil, como componentes en la recuperación de los suelos degradados (Coria y Vega, 2011; Levy, 2007).

La reutilización de las aguas residuales en la agricultura es una forma de reciclar el agua y de purificar los nutrientes (Alvaréz et al., 2006). También, este reuso permite reducir los impactos ambientales a los componentes del agua y al suelo en todo el mundo. É estimado que, el 18\% de las tierras aptas para la agricultura son irrigadas con aguas residuales. Diversas investigaciones muestran, el aprovechamiento de agua de los efluentes residenciales para la irrigación de diferentes cultivos agrícolas (Lado y Bem-Hur, 2009; Mazen et al., 2010; Menegaki et al., 2001; Palese et al., 2009; Qadir et al., 2010; Sousa et al., 2006). De acuerdo con Mazen (2010), la composición media del efluente domestico (sanitario), presenta una mezcla compuesta de agua $(99,9 \%)$ y de sólidos $(0,1 \%)$. Del $70 \%$ de los sólidos encontrados son orgánicos y el 30\% son inorgánicos. Sin embargo, con el transcurrir del tiempo, fue observado una contaminación del suelo, del subsuelo y de las plantas por los del metáis pesados, que son considerados dañinos, por lo tanto disminuyen la calidad de ambos (Al-Hamaiedeh y Bino, 2010; Kiziloglu et al., 2008; Lado y Bem-Hur, 2009; Leal et al., 2010; Menegaki et al., 2007; Qadir, et al., 2010; Rusan et al., 2007; Tose, 2006).

Mas, estudios, también muestran una disminución de perdidas por erosión, una mejora en la estructura física del suelo con el uso de abonos orgánicos, llamados de humos. El uso de abono orgánico, favorece el control biológico. Esto es debido a una mayor presencia microbiana y al aumento de nitrógeno en el suelo. Finalmente, el uso de abonos orgánicos no es tóxico, tienen un menor costo, mejora la absorción de la humedad, tiene mayor fertilidad y aumenta la producción (Mazen et., al. 2010 Santos et al., 2004; Silva et al., 2006). En este contexto, este estudio propone el aprovechamiento de los residuos de la construcción civil (RCC) y el uso de las membranas filtrantes en el tratamiento de efluentes domestico (aguas residuales). Durante este estudio, fue evaluada la eficiencia del RCC en la decantación del lodo del agua residual y el uso de este lodo en la producción de humus. También fue reutilizado los líquidos sobrenadantes de la decantación y de la filtración por medio de las membranas en la irrigación de las semillas de maíz.

\section{MATERIALES Y MÉTODOS}

El efluente sanitario bruto, fue colectado en la salida inicial gradual, proveniente de la estación de tratamiento de aguas residuales (ETE) Anhumas, de la Ciudad de Campinas - São Paulo, Brasil. SANASA es la empresa responsable por la administración y operación de la planta de tratamiento de agua potable y de residuos domésticos. El residuo proveniente de la construcción civil (RCC) fue donado por el Departamento de Ingeniería Civil de la Universidad Estadual de São Paulo, Poli/USP. Las semillas de maíz fueron de la variedad Bandeirante y providenciadas por la CATI/Campinas-São Paulo, Brasil. Finalmente el suelo tiene características arcillosas, de coloración rojo-amarillo, también recolectado en la región de Campinas, São Paulo.

Fue utilizado un planeamiento experimental completo del tipo $2^{3}$. En este planeamiento fue considerado el volumen de RCC a ser usado en la decantación del lodo y la variación de las alturas de las camadas totales del suelo (CTS) en la obtención de los humus. También fue considerado el tiempo de producción del humus, teniendo como respuesta la ganancia de la fertilidad del suelo (ver Tabla 1). Los modelos fueron obtenidos por el método de los mínimos cuadrados. El ajuste matemático fue realizado por el análisis de la varianza (ANOVA) y la optimización del proceso fue realizada por la metodología del análisis de la superficie de 
respuesta (RSM). Ambos métodos fueron utilizados de acuerdo con los estudios de Barros Neto et al. (2007), Biazus et al. (2009), Lopes et al. (2008) y Rodríguez et al. (2008).

El potencial de aplicación de las aguas tratadas en la irrigación, fue comparado con las aguas de abastecimiento (control). En este caso, fue medido el porcentaje de semillas germinadas que estas alcanzan con relación a las semillas germinadas por la irrigación con el agua de control. Las aguas del reuso fueron obtenidas por decantación y después por microfiltración. En escala de laboratorio, fue mezclado $2 \mathrm{~L}$ del efluente domestico, en decantadores con un volumen útil de $3 \mathrm{~L}$. Las membranas fueron del tipo plana, de poli acetato de vinilo, con $707 \mathrm{~mm}^{2}$ de área de filtración aproximadamente y $0,4 \mu \mathrm{m}$ de poros. EI RCC fue usado como un agente de la decantación, en volúmenes diferentes y es presentado en la Tabla 1. Fue precipitada la torta hasta estar totalmente claro el efluente. Fueron consideradas $15 \mathrm{~h}$ de decantación para la evaluación del proceso. A pos esto, fue realizado la separación líquida del sobrenadante, que es la parte sólida (lodo o torta) la cual fue usada en obtención de los humos. El sobrenadante obtenido después de la decantación con el RCC, fue filtrado en la membrana para que sea utilizado como agua de reuso (Lopes et al., 2009; Severo Jr. et al., 2007). DQO, sólidos totales, turbidez y microorganismos (Acosta et al., 2007; Eaton et al., 1995). Después, fueron colocadas en cubas de germinación, el forraje (capin) y el lodo en camadas secuenciales. La secuencia de las camadas fue de: arena, gramíneas, lodo y arena. La variación de las camadas totales del suelo (CTS) en $\mathrm{cm}$. es presentada en la Tabla 2. El proceso de producción de humus fue dejado por un periodo de quince días. Después de formar las camadas se procedió a la irrigación de cada recipiente diariamente y se mixturo las camadas cada dos días. Los ensayos fueron realizados en triplicada (Santos et al., 2004; Silva et al., 2006; Vaz y Gonçalves, 2002; Yamanish et al., 2004). La espectrofotometría de masa con plasma (ICP-MS), fue utilizada para caracterizar las muestras del suelo y compararlas con relación al contenido de sus minerales. Los análisis de ICP-MS, fueron realizadas con equipo Modelo Elan 600. Fue utilizado gases de argón como generador de plasma y una mixtura de $\mathrm{HNO}_{3}$ con $\mathrm{HCl}$ y $\mathrm{HF}$ como solventes (Watanabe et al., 2007).

Finalmente, después de haber terminado el tiempo de producción de humus. Las semillas de maíz fueron sembradas en cada cuba de germinación, con una profundidad de $1 \mathrm{~cm}$., con espaciamiento semejante en todas las plantaciones $(5 \times 5 \mathrm{~cm}$.). Diariamente, las muestras fueron irrigadas con volúmenes iguales de 50 mil de agua. También se adoptó un número igual de semillas para cada experimento (Biazus et al., 2009; Santos et al., 2004; Silva et al., 2006; Yamanish et al., 2004). La ganancia de la fertilidad del suelo por la adición del humus fue medido por la ecuación 1. El porcentaje de semillas germinadas fue obtenido por la ecuación 2. También el rendimiento de agua de reuso fue calculado usando la ecuación 3. Donde: SG es la cantidad de semillas germinadas para la muestra o para el control. STP es el total de las semillas sembradas.

$$
\begin{aligned}
& \text { Fertilidad Ganada (\%) }=\left(\frac{S G_{\text {muestra }}-S G_{\text {Control }}}{S G_{\text {Control }}}\right) * 100 \\
& \% \text { Germinacion }=\left(\frac{S G_{\text {muestra }}}{T S P}\right) * 100 \\
& \text { Rendimient Reuso }(\%)=\left(\frac{S G_{\text {muestra }}}{S G_{\text {Control }}}\right) * 100
\end{aligned}
$$

\section{RESULTADOS Y DISCUSIONES}

Fue observado que después de la adición del RCC al efluente domestico (aguas residuales), la mezcla pasó de una coloración oscura a una coloración amarilla y total decantación fue observada en 5 min. De acuerdo con Severo Jr et al. (2007) Esto es debido a que el material que compone el RCC posee atracción electrostática. Esta atracción favorece la aglomeración de partículas sobre el mismo, generando la formación de flóculos y favorece su precipitación. Este fenómeno observado, permite la clarificación del efluente. El residuo de la construcción civil desempeñó un papel de agregado. En cuanto que, el efluente sanitario sirve como fuente de materia orgánica (carbón, nitrógeno y fósforo). Las gramíneas auxiliaron en la textura y estructuración del suelo, en los experimentos conteniendo los humus. También esta materia orgánica ayudó a controlar la 
humedad del suelo. A medida que el tiempo de producción del humus fue pasando, se observó que la coloración del suelo cambiaba. El color del suelo pasó de rojo para un color más oscuro. Este comportamiento observado, debe estar asociado probablemente a la incorporación de material orgánico en el suelo.

Durante el proceso de germinación de las semillas del maíz, se observo el aparecimiento de brotes después de cinco días. También se observó que la germinación de las semillas fue uniforme. Este crecimiento observado fue más rápido en las cubas conteniendo humus, que en la cuba conteniendo el suelo puro (control). La Tabla 1 presenta los resultados obtenidos sobre la mejora en la fertilidad del suelo. El cálculo de mejora de la fertilidad fue realizado con base en la ecuación 1. Este trabajo obtén $100 \%$ de germinación, en cuanto que otros resultados obtenidos por diversos investigadores mencionado anteriormente, no superaron el 80\% de germinación. Con esta comparación experimental, se puede afirmar que los resultados obtenidos fueron superiores a los valores obtenidos por Sousa et al. (2006), Yamanish et al. (2004) y por Mazon et al. (2010) para el trigo y melón culturas en suelo del desierto con aguas residuales.

La Tabla 2 presenta la evaluación del modelo que más se ajusto a los datos experimentales, por la metodología ANOVA. De acuerdo con Barros Neto et al. (2007) estimó que un método esta ajustado y es significativo estadísticamente si: el valor de $\mathrm{R}^{2}$ fue próximo de 1,0 y los valores de las varianzas fueren $100 \%$. También se considera que él método esta ajustado si el teste $F_{1}$ calculado fuera mayor que el $F_{1}$ tableado y el $F_{2}$ calculado fuera menor que el $F_{2}$ tableado. Como todos estos requisitos fueron completados por el modelo representado en la ecuación 4 . Podemos afirmar que el modelo esta ajustado a los datos experimentales y es estadísticamente significativo. Esta ecuación demuestra que no hay influencia de la camada total del suelo (CTS), sobre la calidad de humus obtenido. Este valor no interfirió en la fertilidad del suelo. De esta forma la optimización fue realizada únicamente con los otros factores, como se ve en la Fig. 1.

Tabla 1. Planeamiento experimental y resultados obtenidos

\begin{tabular}{cccccc}
\hline $\mathrm{N}$ & $\begin{array}{c}\mathrm{RCC} \\
(\mathrm{mL} / \mathrm{L})\end{array}$ & $\begin{array}{c}\text { CTS } \\
(\mathrm{cm} .)\end{array}$ & $\begin{array}{c}\text { Tiempo } \\
(\text { semanas })\end{array}$ & $\begin{array}{c}\text { Germinación } \\
(\%)\end{array}$ & $\begin{array}{c}\text { \% Fertilidad } \\
\text { Ganada }\end{array}$ \\
\hline 1 & 100 & 2 & 1 & 91,28 & 5,56 \\
2 & 100 & 2 & 3 & 94,47 & 9,26 \\
3 & 100 & 4 & 1 & 91,28 & 5,56 \\
4 & 100 & 4 & 3 & 94,47 & 9,26 \\
5 & 200 & 2 & 1 & 91,28 & 5,56 \\
6 & 200 & 2 & 3 & 94,47 & 9,26 \\
7 & 150 & 3 & 2 & 100 & 15,65 \\
8 & 150 & 3 & 2 & 100 & 15,65 \\
9 & 150 & 3 & 2 & 100 & 15,65 \\
10 & 200 & 4 & 1 & 91,28 & 5,56 \\
11 & 200 & 4 & 3 & 94,47 & 9,26 \\
& & Control & & 86,47 & 0,00 \\
\hline
\end{tabular}

Tabla 2. Evaluación del modelo que más se ajusto con una confianza de 95\%

\begin{tabular}{lllll}
\hline Parámetros & Varianza & $\mathrm{R}^{2}$ & $\mathrm{~F}_{1(2,8)}$ & $\mathrm{F}_{2(6,2)}$ \\
Calculado & 100,0 & 1,000 & $7.10^{8}$ & 0,162 \\
Tableado & 100,0 & 1,000 & 3,12 & 9,33 \\
\hline
\end{tabular}

Fertilidad Ganada (\%) $=15,65+1,85$. Tiempo - 8,24.RCC

La Fig. 1 muestra que a medida que se usó cantidades medias de RCC (150ml/L) y un menor tiempo para la producción de humus. La calidad del humus se elevó y surgió un efecto con una mayor fertilidad del suelo estudiado. Yamanish et al. (2004), observó que la inserción de humus o de estiércol a los suelos eleva el grado de germinación. También observó que hay un crecimiento en partes de las plantas de papaya. Esta observación es muy interesante, por que cuando se 
comparó con las plantas de la misma especie que fueron abonadas con abonos comerciales del tipo NPK (nitrógeno, fósforo y potasio), estos valores fueron mejores. Santos et al. (2004) verificaron que el humus obtenidos de materiales como la cáscara de arroz, coco seco y verde, obtuvieron mejores resultados en el crecimiento de plantas de Heliconia psittacorum L., que los humus comerciales. Esto es, debido a que el humus comercial posee vermífugos que son usados para su conservación, más acaba perjudicando su eficiencia en la recuperación de los suelos.

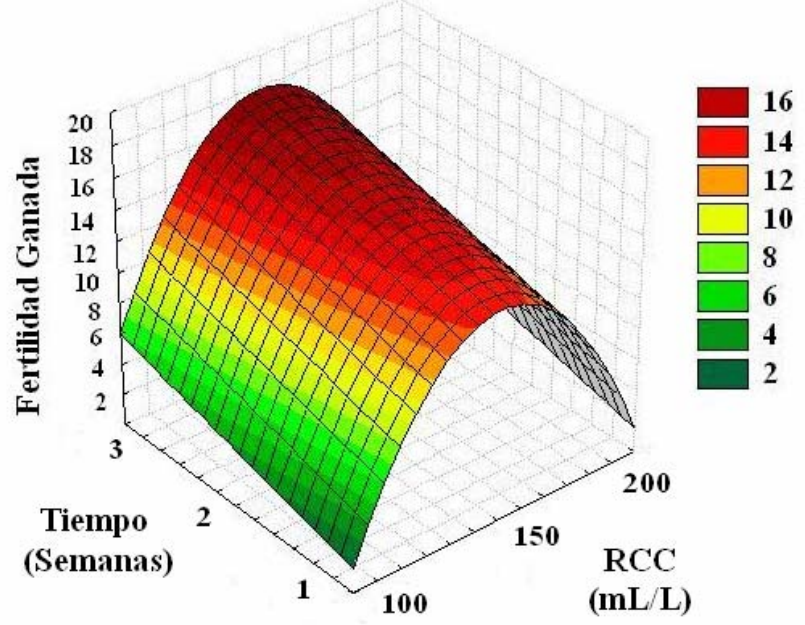

Fig. 1. Superficie de respuesta para evaluar los efectos del RCC y del tiempo de producción de humus sobre la fertilidad del suelo ganado.

La Tabla 3, muestra los resultados obtenidos del análisis de espectrofotometría de masa con plasma del suelo utilizado (control), del suelo conteniendo humus (mejor condición) y del RCC. Cuando comparamos el suelo control con el suelo conteniendo humus es perceptible que hay un enriquecimiento del suelo conteniendo humus. En este suelo fue encontrado potasio, que es uno de los principales nutrientes en la evaluación de la fertilidad de los suelos. Fue posible observar una mayor presencia de micronutriente en este suelo con humus. Fue percibido también que, además de haber la reducción de minerales contaminantes como el hierro, el plomo y la disminución del sodio que es uno de los responsables por la salinidad del suelo. De esta forma, es notoria la mejora la calidad química del suelo con el humus con relación al suelo natural (control) (Al-Hamaiedeh y Bino, 2010; Kiziloglu et al., 2008; Lado y Bem-Hur, 2009; Leal et al., 2010; Menegaki et al., 2007; Rusan et al., 2007; Tose, 2006).

Tabla 3. Resultados del análisis por espectrofotometría de masa con plasma

\begin{tabular}{cccc}
\hline Análisis & Suelo & RCC & humus \\
\hline $\mathrm{Na}(\mathrm{g} / \mathrm{kg})$ & 32,0 & 11,7 & 20,4 \\
$\mathrm{Ca}(\mathrm{g} / \mathrm{kg})$ & 22,7 & 55,1 & 37,7 \\
$\mathrm{Fe}(\mathrm{g} / \mathrm{kg})$ & 63,7 & 18,5 & 13,7 \\
$\mathrm{~K}(\mathrm{~g} / \mathrm{kg})$ & 0,92 & 2,40 & 7,49 \\
$\mathrm{~Pb}(\mathrm{ppm})$ & 41,4 & 89,4 & 0 \\
\hline
\end{tabular}

Las aguas tratadas por membrana tuvieron una turbidez y DQO próximos de $90 \%$. Y también, fue alcanzado el $100 \%$ con la eliminación total de bacterias presentes y sólidos solubles (SS), con relación a las aguas residuales. La Tabla 4 muestra la comparación entre los resultados obtenidos para las muestras de agua de reuso y el control. Fue obtenida una germinación de $81,67 \%$ con agua tratada por membranas filtrantes. Cuando comparamos los rendimientos del agua de reuso con relación al agua del control, se observa que, el agua obtenida por el tratamiento de membranas alcanzó valores próximos del 90\%. Estos valores obtenidos son considerados satisfactorios (Mazen et al., 2010; Sousa et al., 2006; Yamanish et al., 2004). Alvarez et al (2006) destaco en su trabajo la gran disposición de los agricultores a mejorar la gestión del agua y están dispuestos a nuevas tecnologías. El uso de esta agua tratada previene la contaminación microbiana y de los metáis en pesados en la tierra, en el agua, en el subsuelo y en las plantas y reduce el consumo del aguas naturales para las irrigaciones (Al-Hamaiedeh y Bino, 2010; Kiziloglu 
et al., 2008; Lado y Bem-Hur, 2009; Leal et al., 2010; Menegaki et al., 2007; Rusan et al., 2007; Tose, 2006).

Tabla 4. Resultados obtenidos después la irrigación con el agua de reuso

\begin{tabular}{ccc|cccc}
\hline Muestras & \multirow{2}{*}{ \%Germinación } & Rendimiento de & \multicolumn{4}{|c}{ Reducción (\%) } \\
& & agua de reuso (\%) & turbidez & bacterias & DQO & SS \\
\hline CONTROL $_{\text {agua }}$ & $93,33 \pm 5,16$ & $100 \pm 0$ & - & - & - & - \\
MEMBRANA $_{\text {aqua }}$ & $81,67 \pm 5,16$ & $87,50 \pm 2,68$ & $93,3 \pm 0$ & $100 \pm 0$ & $90,1 \pm 0$ & $100 \pm 0$ \\
\hline
\end{tabular}

\section{CONCLUSIONES}

La germinación de las semillas de maíz en los suelos enriquecidos con el humus fue más rápida que en el suelo natural. La mejor condición de contorno fue donde se utilizó el mayor período de tiempo para la producción de humus y cantidades intermedias del RCC. También se verificó un beneficio en la fertilidad del suelo de 15,65\% para la germinación del maíz. Los procesos de separación por membrana y de decantación con el RCC, se mostraron bastante promisorios para ser usado en los tratamientos de efluentes sanitarios. También las aguas tratadas posibilitan el reuso en la irrigación como fue con la cultura del maíz. Esta metodología elimina bacterias, fungos e otros microorganismos causadores de enfermedades patogénicas en las plantas y en el suelo. Esto, también evita la necesidad de hacer un nuevo tratamiento de agua de reuso, para eliminar los microorganismos antes mencionados (ejemplo la cloración química). Se puede concluir algunas ventajas en este trabajo, como la posibilidad de aprovechar el total de las aguas residuales. Esto evitaría el continuo descarte de las aguas residuales a los ríos, por que el tratamiento de agua residual hecho por las ETEs del Brasil son insuficientes y contaminan los ríos. También, se evitaría disminuir los gastos con aguas captadas de los diversos cuerpos hídricos para su uso en la agricultura.

\section{AGRADECIMIENTOS}

A UNINOVE por el apoyo económico en lo desarrollo de la pesquisa y a SANASA por el efluente domestico.

\section{REFERENCIAS}

Acosta, I., M. G. Moctezuma-Zárate, J. F. Cárdenas y C. Gutiérrez, Bioadsorción de Cadmio (II) en Solución Acuosa por Biomasas Fúngicas, Información Tecnológica: 18(1), 9-14 (2007)

Al-Hamaiedeh, H. y M. Bino, Effect of treated grey water reuse in irrigation on soil and plants, Desalination: 256 (1-3), 115-119 (2010).

Álvarez, C. J., T. S. Cuesta, J. J. Cancela y M. F. Marey, Gestión de Aguas Residuales en el Ámbito Rural en Galicia, España, Información Tecnológica: 17(3), 15-21 (2006).

Barros Neto, B.; Scarminio, I.S.; Bruns, R.E., Como Fazer Experimentos: Pesquisa e Desenvolvimento na Ciência e na Indústria, $3^{\mathrm{a}}$ edición, Editora da Universidade Estadual de Campinas, Campinas, São Paulo, Brasil (2007).

Biazus, J.P.M., R.R. Souza, J.E. Marquez, T.T. Franco, J.C.C. Santana, E.B. Tambourgi, Production and characterization of amylases from Zea mays malt, Brazilian Archive of Biology and Technology: 52(4), 991-1000 (2009).

Coria, I.D. y Vegas, M., Determinación de la Efectividad de la Remediación de Suelos Accidentalmente Contaminados con Ácido Sulfúrico mediante Métodos Geofísicos, Información Tecnológica: 22(1), 3-8 (2011). 
Eaton, A.D., L.S. Clesceri, A.E. Greenberg, Standard methods for the examination of water and wasterwater. 19th edition, American Public Health Association, Washington, USA (1995).

Kiziloglu, F. M.; M. Turan, U. Sahin, Y. Kuslu y A. Dursun, Effects of untreated and treated wastewater irrigation on some chemical properties of cauliflower (Brassica olerecea L. var. botrytis) and red cabbage (Brassica olerecea L. var. rubra) grown on calcareous soil in Turkey, Agricultural Water Management: 95(6), 716-724 (2008).

Lado, M. y M. Bem-Hur, M. Treated domestic sewage irrigation effects on soil hydraulic properties in arid and semiarid zones: A review, Soil and Tillage Research: 106(1), 152-163 (2009).

Leal, R. M. P.; L. P. Firme, U. Herpin, A. F. Fonseca, C. R. Montes, C. T. S. Dias y A. J. Melfi, Carbon and nitrogen cycling in a tropical Brazilian soil cropped with sugarcane and irrigated with wastewater, Agricultural Water Management: 97(2), 271-276 (2010).

Levy, S. M. Sustentabilidade na construção civil - a contribuição do concreto. In: Concreto: Ensino, Pesquisa e Realizações, Editora do Instituto Brasileiro do Concreto, São Paulo, Brasil (2007).

Lopes, F.L.G., J.B. Severo Júnior, R.R. Souza, D.D. Ehrhardt, J.C.C. Santana y E.B. Tambourgi, Concentration by Membrane Separation Processes of a Medicinal Product Obtained from Pineapple Pulp, Brazilian Archive of Biology and Technology: 52(2), 457-464 (2009).

Menegaki, A. N., N. Hanley y K. P. Tsagarakis, The social acceptability and valuation of recycled water in Crete: A study of consumers' and farmers' attitudes, Ecological Economics: 62(1), 7-18 (2007).

Mazen, A.; F.A. Faheed y A.F. Ahmed, Study of potential impacts of using sewage sludge in the amendment of desert reclaimed soil on wheat and jews mallow plants, Brazilian Archive of Biology and Technology: 53(4), 917-930 (2010).

Palese, A. M., V. Pasquale, G. Celano, G. Figliuolo, S. Masi y C. Xiloyannis, Irrigation of olive groves in Southern Italy with treated municipal wastewater: Effects on microbiological quality of soil and fruits, Agriculture, Ecosystems \& Environment: 129 (1-3), 43-51 (2009).

Qadir, M., D. Wichelns, L. Raschid-Sally, P. G. McCornick, P. Drechsel, A. Bahri y P.S. Minhas, The challenges of wastewater irrigation in developing countries, Agricultural Water Management: 97(4), 561-568 (2010).

Rodríguez, M. E., R. C. Miranda, R. Olivas y C. A. Sosa, Efectos de las Condiciones de Operación Sobre La Biosorción de Pb2+, Cd2+ y Cr3+ en Solución por Saccharomyces cerevisiae Residual. Información Tecnológica: 19(6), 47-55 (2008).

Rusan, M. J. M., S. Hinnawi y L. Rousan, Long term effect of wastewater irrigation of forage crops on soil and plant quality parameters, Desalination: 215(1-3), 143-152 (2007).

Santos, M.R.A., A.L.O. Timbó, A.C.P.P. Carvalho y J.P.S. Moraes, Avaliação de substratos e adubos orgânicos na aclimatização de plântulas de Heliconia psittacorum, Pesquisa Agropecuária Brasileira: 39(10),1049-1051 (2004).

Severo Jr., J.B.; S.S. Almeida, N. Narain, R.R. Souza, J.C.C. Santana y E.B. Tambourgi, Wine clarification from Spondias mombin L. pulp by hollow fiber membrane system, Process Biochemistry: 42(11), 1516-1520 (2007).

Silva, J.C., C.C. Costa, C. Duda, P.C. Timossi y C.I. Leite, Crescimento e produção de rabanete cultivado com diferentes doses de húmus de minhoca e esterco bovino, Ceres: 53(305), 25-30 (2006). 
Sousa, J.T., B.S.O. Ceballos, J.P. Dantas, I.N. Henrique y S.M.S. Lima, Reúso de água residuária na produção de pimentão (Annuum L.). Revista Brasileira Engenharia Agrícola e Ambiental: 10(1), 89-96 (2006).

Tose, S, Reuse of effluent water - benefits and risks, Agricultural Water Management: 80 (1-3), 147-159 (2006).

Watanabe, S., T.M.B. Farias, R.F. Gennari, G.M. Ferraz y R. Kunzli, Chemical Process to separate Iron Oxides Particles in Pottery Sample for EPR Dating, Spectrochimica Acta. Part A, Molecular and Biomolecular Spectroscopy: 10, 10-18 (2007).

Yamanishi, O.K., G.R. Fagundes, J.A. Machado Filho y G.V. Valone, Efeito de diferentes substratos e duas formas de adubação na produção de mudas de mamoeiro, Revista Brasileira de Fruticultura: 26(2), 276-279 (2004). 